\title{
Anisotropic quantum dots: Correspondence between quantum and classical Wigner molecules, parity symmetry, and broken-symmetry states
}

\author{
B. Szafran, ${ }^{1,2}$ F. M. Peeters, ${ }^{1}$ S. Bednarek, ${ }^{2}$ and J. Adamowski ${ }^{2}$ \\ ${ }^{1}$ Departement Natuurkunde, Universiteit Antwerpen (Campus Drie Eiken), B-2610 Antwerpen, Belgium \\ ${ }^{2}$ Faculty of Physics and Nuclear Techniques, AGH University of Science and Technology, al. Mickiewicza 30, 30-059 Kraków, Poland
}

(Received 7 November 2003; published 30 March 2004)

\begin{abstract}
We study electron systems confined in anisotropic quantum dots at high magnetic fields using the configuration-interaction scheme with a multicenter basis of single-electron functions centered around different sites. Elliptical, triangular, and square quantum dots are investigated. We study the relation between the quantum and classical charge density and conclude that at high magnetic field the quantum charge density reproduces all the equivalent lowest-energy configurations of classical point charges. Quantum systems with a classical counterpart of a unique lowest-energy configuration exhibit a smooth convergence of the charge density to the classical limit at high magnetic field. In quantum systems with several equivalent classical configurations the magnetic field induces discontinuous transformations of the ground-state symmetry associated with crossings of the corresponding few-electron energy levels. A linear combination of states with the crossing levels yields a semiclassical charge density with a broken symmetry. At the magnetic field corresponding to the level crossing this combination is an exact eigenstate of the Hamiltonian. For circular dots the present findings give an additional insight into the properties of the magic-angular-momenta states and into the physics behind the broken-symmetry mean-field solutions.
\end{abstract}

DOI: 10.1103/PhysRevB.69.125344

PACS number(s): 73.21.-b, 73.22.Gk

\section{INTRODUCTION}

Quantum dots $^{1}$ provide a convenient testing ground for studying electron localization in potentials which can be, to a certain extent, formed at will by proper etching techniques or chosen geometry of the applied gate electrodes. One of the most interesting problems in this field is the Wigner crystallization of the electron system induced by a high magnetic field. The problem of Wigner crystallization in cylindrical quantum dots ${ }^{2-4}$ has been widely discussed and at present seems to be well understood both quantum mechanically and classically. ${ }^{5}$ Wigner crystallization, i.e., the separation of electrons, in cylindrically symmetric potential appears in the inner coordinates of the system and the charges of separate electrons are not distinguishable in the rotationally invariant electron density. Therefore, in cylindrical quantum dots methods ${ }^{6}$ based on charge-density measurements are not appropriate for the observation of Wigner crystallization. However, such an approach is possible in structures of lower symmetry. Previous exact diagonalization studies of a threeelectron system in a triangular quantum $\operatorname{dot}^{7}$ and of a system of six electrons in an elliptical quantum $\operatorname{dot}^{8}$ showed that Wigner crystallization can be observed in the charge-density distribution of the electrons in the laboratory frame. Moreover, an exact study of the two-electron system has been presented $^{9}$ for triangular, square, and hexagonal quantum dots in the absence of a magnetic field and in an elliptical dot at zero magnetic field. ${ }^{10}$ Wigner crystallization of fewelectron systems in large polygonal quantum dots in the absence of an external magnetic field was studied using density-functional theory. ${ }^{11-13}$ This theory was also applied to evaluate the addition spectra in elliptical quantum dots. ${ }^{14}$ The addition spectra of elliptical dots have been studied with Hartree-Fock method ${ }^{15}$ as well. The effect of pinning of the
Wigner molecule by a Gaussian impurity perturbation in an isotropic confinement potential was recently studied by the quantum Monte Carlo approach. ${ }^{16,17}$

In this paper we perform a detailed study of the charge density of two-, three-, and four-electron systems confined in anisotropic potentials using an exact diagonalization approach. We consider elliptical, square, and triangular shaped quantum dots and investigate the magnetic-field-induced Wigner crystallization. A relation between the quantum and classical charge distributions in anisotropic structures ${ }^{18}$ is found. In particular we discuss the high-magnetic-field behavior of quantum systems whose classical counterparts possess several equivalent lowest-energy configurations.

A lot of attention was paid ${ }^{2,3}$ to the problem of the sequence of the ground-state angular momenta after the maximum density droplet ${ }^{19}$ (MDD) breakdown in cylindrical quantum dots. The magnetic field increases the absolute value of the angular momentum of the confined electron system, but only certain angular momenta with magic quantum numbers ${ }^{2,3,20-22}$ are realized. For the magic angular momenta the classical symmetry is reproduced in the inner coordinates of the quantum system ${ }^{2}$ and the electron-electron interaction energy as a function of the angular momentum presents local minima. ${ }^{23}$ At the end of this paper we point out a relation between the magic angular momenta and the charge density in the laboratory frame. We also consider the magnetic-fieldinduced parity transformations in elliptical dots which are less thoroughly studied counterparts of the angularmomentum transitions appearing in circular dots.

To discuss the charge-density distribution the application of an exact diagonalization method is crucial since meanfield approaches may lead to an artifactal breaking of the symmetry of the confinement potential. ${ }^{3}$ The brokensymmetry solutions present a semiclassical type of localization. On the other hand we found ${ }^{24}$ recently that in the 
strictly infinite magnetic-field limit the energy of the brokensymmetry solution with semiclassical localization becomes equal to the energy of the exact solution. In this paper we study the realistic case of high but finite magnetic fields and extend our previous work to anisotropic confinement potentials. In particular, we reconsider the problem of the symmetry breaking at the exact diagonalization level and demonstrate that a construction of the exact broken-symmetry solution of the few-electron Schrödinger equation is possible for certain values of the magnetic field.

Some of the previous studies of Wigner crystals ${ }^{25-27}$ and Wigner molecules ${ }^{23,24,28,29}$ used a multicenter basis of the single-electron lowest-Landau-level functions. However, these calculations ${ }^{23-29}$ used Hartree-Fock or similar approaches. In the present paper, we use the single-electron multicenter basis for the construction of the configurationinteraction approach which allows for an exact solution of the Schrödinger equation for few-electron systems confined in potentials of arbitrary symmetry. This approach is a generalization of the single-configurational multicenter HarteeFock (MCHF) method ${ }^{24,29}$ which we elaborated previously.

The paper is organized as follows. The second section describes the multicenter-configuration-interaction method as applied in this paper as well as presents test calculations for circular dots. Sections III, IV, and V contain discussion of results obtained for elliptical, square, and triangular dots, respectively. The conclusions reached for lower-symmetry structures are discussed in the context of circular dots in Sec. VI. Summary and conclusions are given in Sec. VII.

\section{MULTICENTER-CONFIGURATION-INTERACTION METHOD}

We consider $N$ electrons confined in a two-dimensional ( $x, y$-plane) quantum dot subject to a strong external magnetic field oriented parallel to the $z$ axis. We solve the $N$-electron Schrödinger equation with the Hamiltonian

$$
H=\sum_{i=1}^{N} h_{i}+\sum_{i=1}^{N} \sum_{j>i}^{N} \frac{\kappa}{r_{i j}}+B S_{z} g^{*} \mu_{B},
$$

where $h$ stands for the single-electron Hamiltonian,

$$
h=\frac{1}{2 m^{*}}(-i \hbar \boldsymbol{\nabla}+e \mathbf{A})^{2}+V(x, y),
$$

$g^{*}$ is the effective Lande factor, $S_{z}$ the $z$ component of the total spin, $B$ the magnetic field, $\mu_{B}$ stands for the Bohr magneton, $\kappa=e^{2} / 4 \pi \epsilon_{0} \epsilon, \epsilon$ is the dielectric constant and $m^{*}$ the electron effective mass. We use the Landau gauge A $=(-B y, 0,0)$ and adopt material parameters for GaAs, i.e., $m * / m_{0}=0.067, \epsilon=12.9$, and $g^{*}=-0.44$.

We assume complete spin polarization of the electron system by the external magnetic field. The multicenterconfiguration-interaction (MCI) scheme is constructed in the following way. First we diagonalize the single-electron Hamiltonian (2) in a multicenter basis

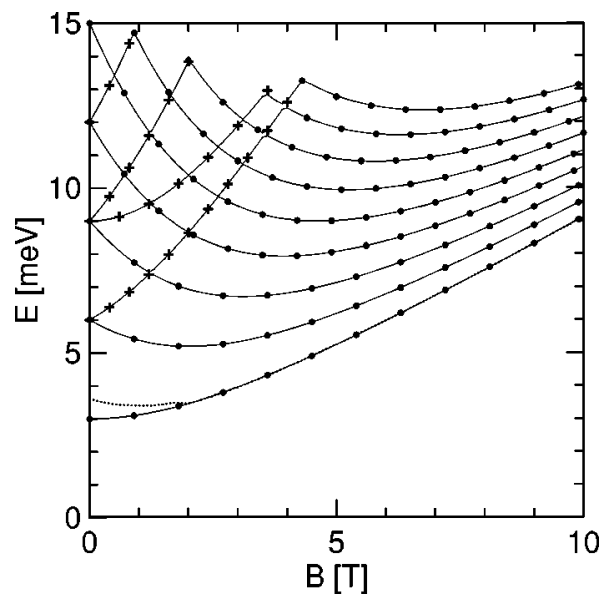

FIG. 1. The single-electron spectrum calculated with the trial wave function (3) for an isotropic quantum dot with $\hbar \omega=3 \mathrm{meV}$ (solid lines). Eleven centers have been used, one located at the origin and the others at the circumference of a circle with variationally optimized radius. Symbols show the exact Fock-Darwin energy levels corresponding to the lowest (dots) and higher bands (crosses). The dotted line shows the estimate for the ground-state energy obtained when the center located at the origin is excluded from the basis.

$$
\Psi_{\mu}(\mathbf{r})=\sum_{i=1}^{M} c_{i}^{\mu} \psi_{\mathbf{R}_{i}}(\mathbf{r})
$$

with

$$
\psi_{\mathbf{R}}(\mathbf{r})=\sqrt{\frac{\alpha}{2 \pi}} \exp \left\{-\frac{\alpha}{4}(\mathbf{r}-\mathbf{R})^{2}+\frac{i \beta}{2}(x-X)(y+Y)\right\},
$$

where $M \geqslant N$ is the number of centers $\mathbf{R}=(X, Y)$. Next, the $N$-electron Hamiltonian (1) is diagonalized in a basis of $M ! / N !(M-N)$ ! Slater determinants constructed from the single-electron orthonormal eigenvectors $\Psi_{\mu}$ (3) with $\mu$ $=1, \ldots, M$. The position of the centers $\mathbf{R}_{i}, \alpha$, and $\beta$ are nonlinear variational parameters optimized for the total energy of the $N$-electron system. For three or more centers per electron the optimal value of the parameter $\beta$ tends to $e B / \hbar$. At high magnetic field the parameter $\alpha$ also takes this value independently of $M$. For $\alpha=\beta=e B / \hbar$ the wave function (4) is the lowest-Landau-level eigenfunction.

The flexibility of the single-electron basis was verified for an isotropic parabolic potential $V(x, y)=m^{*} \omega^{2}\left(x^{2}+y^{2}\right) / 2$, with $\hbar \omega=3 \mathrm{meV}$. Solid lines in Fig. 1 show the magneticfield dependence of the single-electron spectrum calculated with the trial wave function (3) with ten centers located at the circumference of a circle with equal angular spacings and the 11th center located at the origin. The radius of the circle was optimized variationally. The dots in Fig. 1 show the exact Fock-Darwin energy levels corresponding to the lowest band which at high magnetic field converges to the lowest Landau level. The crosses mark the energies of the higher Fock-Darwin bands. The present calculations with wave function (3) reproduce the exact single-electron spectrum 
with a high precision. It is interesting to note that the basis (3) constructed of the displaced lowest-Landau-level wave functions (4) reproduces also higher Fock-Darwin bands. Since we are using the Landau gauge the single-electron wave functions (3) are not eigenfunctions of the angularmomentum operator. However, for all the plots presented in Fig. 1, the expectation values of the angular momentum calculated for the wave functions (3) reproduce exactly the correct eigenvalues.

The exact ground Fock-Darwin state wave function possesses the form (4) with $X=Y=0$. The dotted line in Fig. 1 shows the variational estimate of the ground-state energy obtained when the center at the origin is excluded from the basis. The related overestimation of the ground-state energy is nonzero only at low magnetic field $(B \lesssim 2.2 \mathrm{~T})$. We have found that the center located at the origin has no influence on the energy estimates for the single-electron states with nonzero angular momentum. Its contribution to the energy of the excited $s$ states tends to zero at higher magnetic field similarly as for the ground state.

We have performed further tests for the isotropic harmonic confinement potential in order to verify the reliability of the present configuration-interaction approach to the fewparticle states at high magnetic field. The results were compared with the standard exact diagonalization method with a basis constructed from the Fock-Darwin states of definite angular momentum. ${ }^{24,30}$ The reference method ${ }^{24,30}$ assumes neither spin polarization nor the occupation of the lowest Landau level and for $N \leqslant 4$ allows for nearly exact evaluation of the total energy.

Figure 2(a) shows the comparison of the exact total angular momentum of the three-electron system and the expectation values obtained with the MCI wave function for various number of centers placed on a circumference of a circle with equal angular spacings. The plot starts for $B=3 \mathrm{~T}$ for which the ground state is the spin-polarized MDD. ${ }^{19}$ The MDD decays at $B=4.6 \mathrm{~T}$ [cf. black dots in Fig. 2(a)] to a nonpolarized state with angular momentum $-5 \hbar$. For $B>5.4 \mathrm{~T}$ the ground state of the three-electron system is spin polarized. Then, the angular momentum takes the magic values $^{2,3,20-22}$ and changes by $3 \hbar$ as the magnetic field increases.

The present method with $M=N$ uses only a single configuration and at high magnetic field is equivalent to the multicenter HF method. ${ }^{29}$ For $M=N=3$ the MCI method reproduces the angular momentum of the MDD state $[\mathrm{cf}$. squares in Fig. 2(a)]. After the MDD decay, the expectation value decreases linearly with $B$ in contrast to the exact stepwise decrease. The MCI method with six centers reproduces also the exact angular momentum of the first spin-polarized state after the MDD decay, and at higher magnetic fields it starts to decrease linearly with $B$. For $M=12$ the MCI method yields correct expectation values of the angular momenta of all spin-polarized states in the entire considered range of magnetic fields.

Let us now discuss the convergence of the energy estimates obtained with the MCI method to the exact groundstate energy [cf. Fig. 2(b)]. The overestimation of the exact energy with a single configuration $[M=N=3$, cf. the upper-

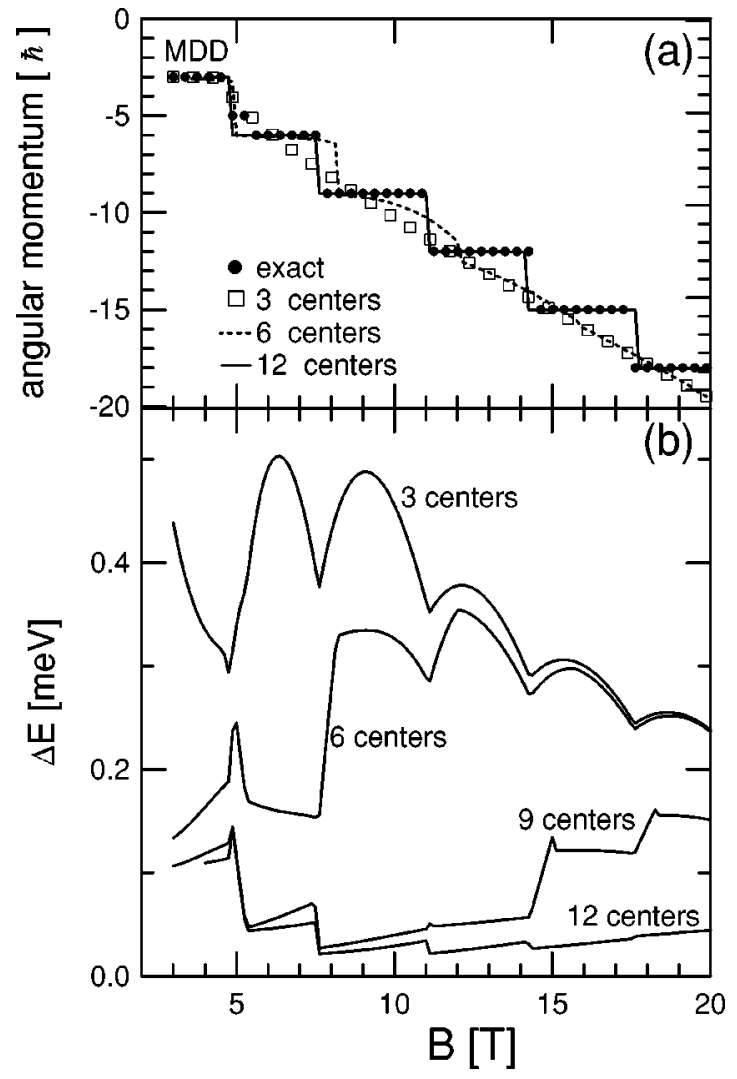

FIG. 2. (a) Exact total angular momentum of the ground state of the three-electron system confined in an isotropic quantum dot with $\hbar \omega=3 \mathrm{meV}$ and the expectation values for the multicenterconfiguration-interaction wave functions using various number of centers. (b) The overestimation of the total three-electron energy with the multicenter approach for different number of centers.

most curve of Fig. 2(b)] is a nonmonotonous function of $B$. The oscillations are due to the fact that the energy estimate of MCI with $M=N$ is a smooth function of $B$, while the exact energy exhibits cusps at these values of $B$ for which the ground-state angular momentum changes. The envelope of these oscillations decreases to 0 in the infinite magnetic-field limit. ${ }^{24}$ Introduction of six centers reduces the overestimation of the total energy as long as the total angular momentum is reproduced by the MCI method with $M=6$ [cf. dashed line in the upper panel of Fig. 2(a)]. At higher magnetic field, for which the overlaps of functions (4) centered around different sites vanish, the precision of the MCI method with $M=6$ deteriorates to the one obtained with $M=3$, i.e., to the MCHF method. ${ }^{29}$ The method with $M=9$ (12) centers gives the exact energy to a precision better than $0.15 \mathrm{meV}(0.05$ $\mathrm{meV}$ ) for $5.4<B<20 \mathrm{~T}$, i.e., in the studied range of magnetic field after the MDD breakdown for which the adopted assumption of spin polarization is fulfilled. Results of similar test calculations for $N=2$ and 4 show that the corresponding upper bounds for the precision of the MCI method with three centers per electron $(M / N=3)$ equal 0.1 and $0.12 \mathrm{meV}$, respectively.

Figure 3 shows the charge density of the three-electron system at $B=6 \mathrm{~T}$ for three, six, and nine centers. In the single-configuration charge density $(M=3)$ the circular 


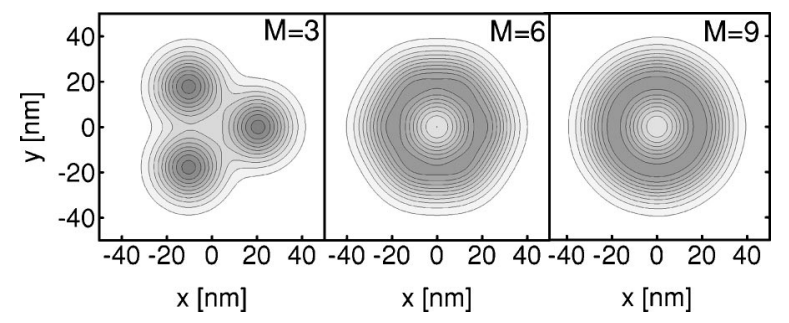

FIG. 3. MCI charge density obtained for the three-electron system with different $M$ for $\hbar \omega=3 \mathrm{meV}$ and $B=6 \mathrm{~T}$. The darker the shade of gray the larger the electron density.

symmetry of the external potential is broken. For six centers the cylindrical symmetry is approximately restored, although at a closer inspection the sixfold symmetry-a trace of the choice of centers - can be noticed. For $M=9$ the charge density shows a perfect cylindrical symmetry. The present numerical method restores the cylindrical symmetry in a manner alternative to the rotated-electron-molecule approach of Yannouleas and Landman. ${ }^{23}$

We conclude that the single-electron wave functions (3) used in the present MCI approach work similarly as the Fock-Darwin functions with definite angular momentum (cf. Figs. 1 and 2). At high magnetic fields the precision of the MCI method is not worse than the MCHF method ${ }^{29}$ which in turn gives exact results in the infinite magnetic-field limit. ${ }^{24}$ The applicability of the present configuration-interaction approach is not limited to cylindrically symmetric potentials. The MCI method allows for a flexible choice of the position of centers which can be tailored to any smooth external potential of arbitrary profile and symmetry.

\section{ELLIPTICAL QUANTUM DOT}

\section{A. Wigner crystallization}

In this section we applied the MCI method to analyze Wigner crystallization in elliptical quantum dots with anisotropic parabolic potential $V(x, y)=m^{*}\left(\omega_{x}^{2} x^{2}+\omega_{y}^{2} y^{2}\right) / 2$ with $\omega_{y}>\omega_{x}$. Calculations were performed with $M=12$ centers put on an ellipse symmetric with respect to the $x$ and $y$ axes with equal angular spacings.

Figure 4 shows the calculated charge densities for two-, three-, and four-electron systems at different magnetic fields (the energies obtained are listed in Table I). The lowest panel shows the position of the classical point charges in the lowest-energy configurations. The classical two- and fourelectron systems in the studied potential possess a unique lowest-energy configuration. On the other hand the classical system of three electrons possesses two equivalent configurations (marked by "black" and "white" symbols in the lowest panel of Fig. 4). Existence of several equivalent classical configurations will be referred to as classical degeneracy.

At relatively low magnetic field $(4 \mathrm{~T})$ the electron puddles look very similar and exhibit two maxima at their left and right $(x)$ ends. For $B=8 \mathrm{~T}$ the electrons in the two- and four-electron systems start to become spatially separated. At higher magnetic field the electron charge densities for $N$ $=2$ and 4 tend to the charge distributions of their classical

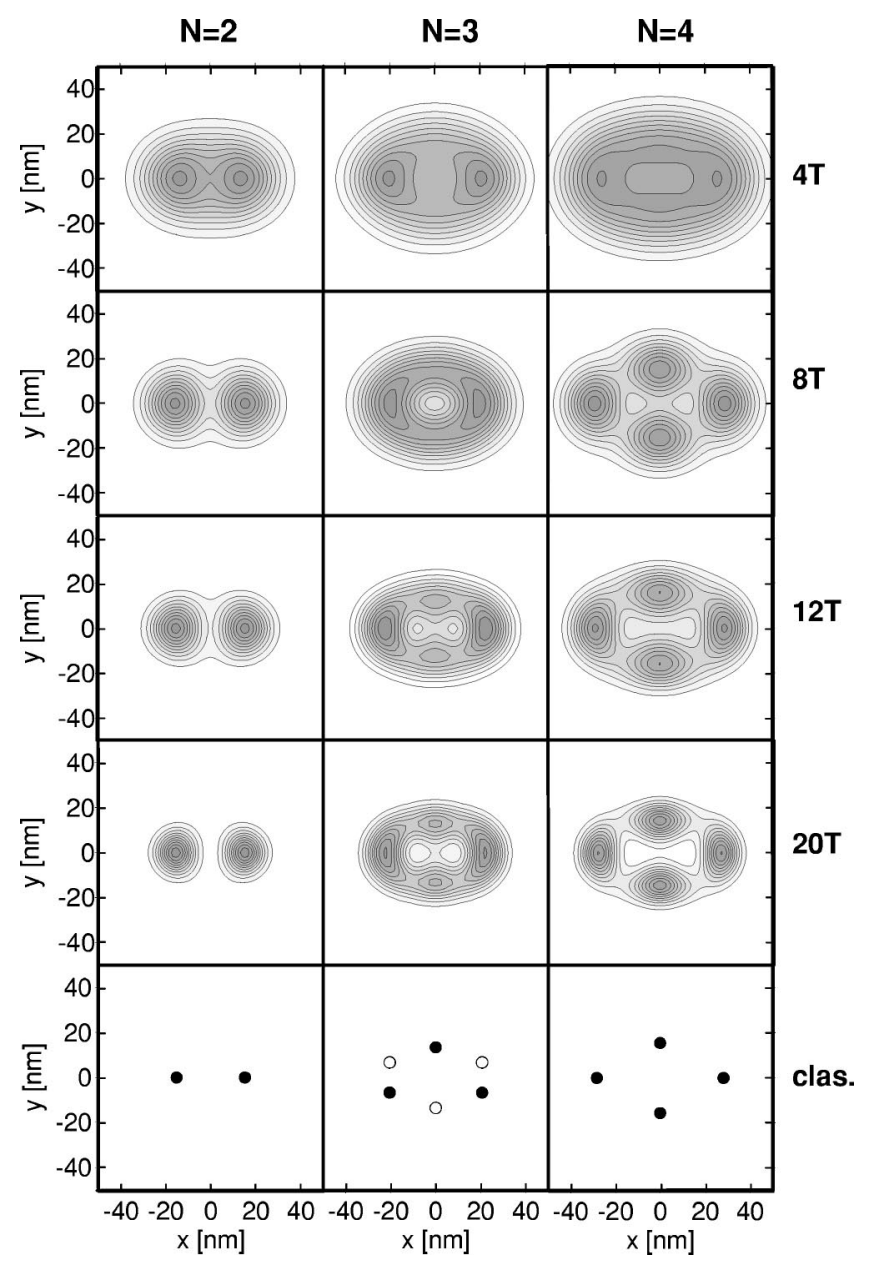

FIG. 4. Charge density of two-, three-, and four-electron systems for an anisotropic parabolic potential with $\hbar \omega_{x}=3 \mathrm{meV}$ and $\hbar \omega_{y}=4 \mathrm{meV}$ for different magnetic fields. The lowest panel presents the lowest-energy configurations of the classical point-charge systems. For $N=3$ the two energy-equivalent configurations are marked with full and open circles.

counterparts (cf. lowest panel of Fig. 4), which results from the shrinkage of the Landau radius with growing magnetic field. For $B=8 \mathrm{~T}$ a central hole in the three-electron charge density appears. The plots of the three-electron charge densities for 12 and $20 \mathrm{~T}$ (cf. Fig. 4) show an appearance of two smaller maxima of the charge density along the $y$ axis. This is shown more clearly in Fig. 5 for the three-electron charge density at $B=30 \mathrm{~T}$. The two charge maxima at the $x$ ex-

TABLE I. Total energy of the $N$-electron system $E_{N}$ (in meV) confined in an anisotropic parabolic potential with $\hbar \omega_{x}=3 \mathrm{meV}$ and $\hbar \omega_{y}=4 \mathrm{meV}$.

\begin{tabular}{cccc}
\hline \hline$B(\mathrm{~T})$ & $E_{2}$ & $E_{3}$ & $E_{4}$ \\
\hline 4 & 15.35 & 30.33 & 49.29 \\
8 & 20.99 & 39.02 & 60.71 \\
12 & 27.20 & 48.20 & 72.94 \\
16 & 33.71 & 58.04 & 85.95 \\
20 & 40.33 & 67.95 & 99.29 \\
\hline \hline
\end{tabular}




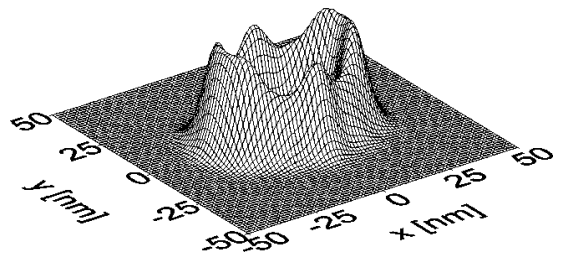

FIG. 5. Charge density of the three-electron system for an anisotropic parabolic potential with $\hbar \omega_{x}=3 \mathrm{meV}, \hbar \omega_{y}=4 \mathrm{meV}$, and $B=30 \mathrm{~T}$.

tremities of the charge puddle are spread out along the $y$ direction into two distinct maxima. The results of Figs. 4 and 5 show that the density distribution for the system of three electrons at high magnetic field tends to a linear combination of the two distributions of the degenerate classical configurations (cf. the lowest panel of Fig. 4).

Evidence of Wigner crystallization in the four-electron charge density is not always as apparent as in the case shown in Fig. 4. The left panel of Fig. 6 displays the charge density of the four-electron system at $B=20 \mathrm{~T}$ for $\hbar \omega_{x}=3 \mathrm{meV}$ and different $\hbar \omega_{y}$. The right panel of the figure shows the corresponding configurations of the classical system. ${ }^{18}$ The clas-

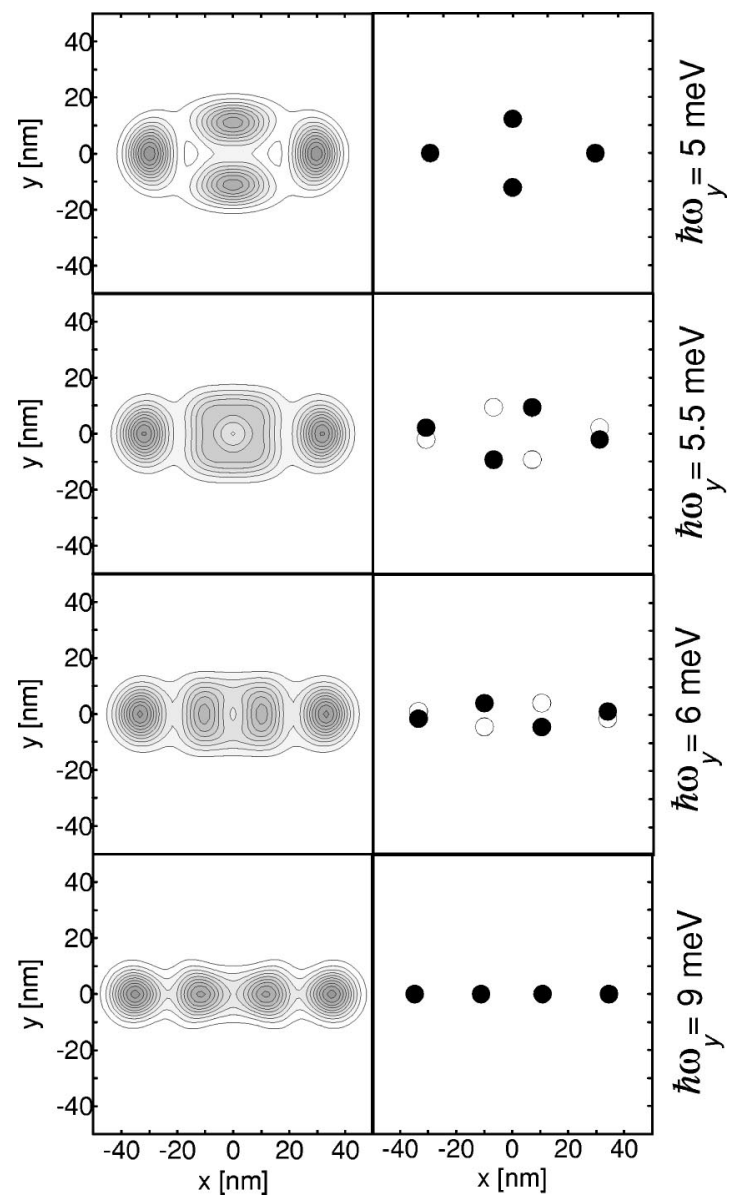

FIG. 6. Left panel: Charge density of the four-electron system at $B=20 \mathrm{~T}$ for $\hbar \omega_{x}=3 \mathrm{meV}$ and different values of $\hbar \omega_{y}$. Right panel: Classical lowest-energy configurations. For $\hbar \omega_{y}=5.5$ and $6 \mathrm{meV}$ two equivalent configurations are marked with different symbols. sical system possesses a single lowest-energy configuration for $3 \mathrm{meV}<\hbar \omega_{y} \lesssim 5.1 \mathrm{meV}$ with electrons situated along the $x$ and $y$ axes. For $\hbar \omega_{y}$ larger than $5.1 \mathrm{meV}$ the electrons leave the axes and as a consequence two equivalent configurations appear. As $\hbar \omega_{y}$ is increased further the classical electrons become localized on the $x$ axis and the classical degeneracy is removed. Note that the classical system exhibits a zigzag transition as discussed in more detail in Ref. 18. The classical configurations and the quantum charge distribution (cf. left panel of Fig. 6) are clearly related. In the absence of classical degeneracy $\left(\hbar \omega_{y}=5\right.$ and $\left.9 \mathrm{meV}\right)$ the quantum charge density possesses four nearly equal maxima localized close to the positions of the classical electrons in equilibrium. The plot for $\hbar \omega_{y}=9 \mathrm{meV}$ represents a nearly onedimensional case of a Wigner molecule, ${ }^{31}$ in which the charge maxima at the ends of the puddle are slightly more pronounced than the maxima in its interior. A trace of the classical degeneracy for $\hbar \omega_{y}=6 \mathrm{meV}$ in the quantum charge density is the elongation of the central maxima in the $y$ direction. These central maxima for $\hbar \omega_{y}=5.5 \mathrm{meV}$ merge into a single ringlike plateau with a hole in the center. For this special case the separation of electrons is not complete, since the charges of the two central electrons occupy the same island. We have found that in this case the separation of the two electrons cannot be observed even in the pair-correlation function $^{2}$ plots. Therefore, the four-electron system in this potential for $B=20 \mathrm{~T}$ presents an interesting case of partial Wigner crystallization.

\section{B. Parity symmetry}

Let us now consider the spatial symmetry of the fewelectron wave function in an elliptical dot. In an anisotropic confinement potential the angular momentum is no longer quantized. However, the wave functions of the few-electron systems in an elliptical dot have a definite parity with respect to the rotation by $\pi$ angle. In cylindrical quantum dots the parity of the states is even (odd) if the angular momentum is an even (odd) multiple of $\hbar$. The MDD states for two, three, and four electrons have angular momentum equal to $-\hbar$, $-3 \hbar$, and $-6 \hbar$, respectively. Therefore, the two- and threeelectron MDD states are of odd parity and the four-electron MDD state is of even parity. At magnetic fields above the MDD breakdown the angular momentum of the ground state takes the magic values ${ }^{2,3,20-22}$ and changes by $N \hbar$. As a consequence the two- and four-electron systems in the MDD state and after its decay possess always the same parity (odd for $N=2$ and even for $N=4$ ), while for three electrons the parity changes with each ground-state transformation.

Solid lines in Fig. 7(a) show the two lowest-energy twoelectron levels in the magnetic-field range corresponding to the MDD decay in a circular quantum dot. The displayed energy $E^{\prime}$ is calculated with respect to the lowest Landau level, i.e., $E^{\prime}=E-N \hbar\left(\omega_{c}+S_{z} g^{*} \mu_{B} B\right)=E-0.85(\mathrm{meV} / \mathrm{T})$ $\times N B$. The two-electron MDD decays at $B=5.75 \mathrm{~T}$ as the energy of the state with angular momentum $-3 \hbar$ crosses the MDD energy level with $L=-\hbar$. The dashed lines present the magnetic-field dependence of the two lowest levels for an elliptical quantum dot with $\hbar \omega_{x}=3 \mathrm{meV}$ and $\hbar \omega_{y}$ 

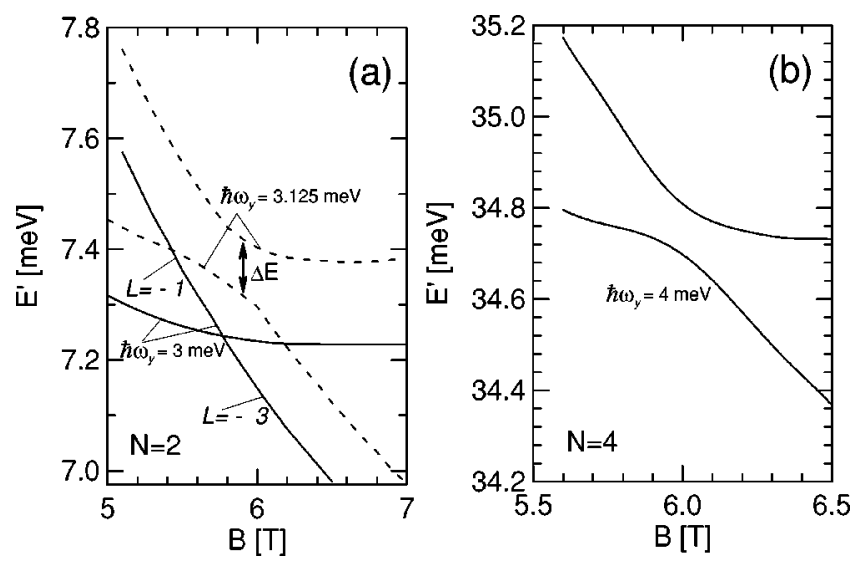

FIG. 7. Two lowest-energy levels of two (a) and four (b) electrons for $\hbar \omega_{x}=3 \mathrm{meV}$ calculated with respect to the lowest Landau level as functions of the magnetic field.

$=3.125 \mathrm{meV}$. Instead of a level crossing we observe an avoided crossing. The avoided crossing is due to the fact that the states involved are of the same odd parity. A similar avoided crossing related to the MDD decay in elliptical dot is observed for four electrons [cf. Fig. 7(b)]. Both the energy levels presented in Fig. 7(b) correspond to even parity states. For both $N=2$ and 4 , further avoided crossings appear at higher magnetic fields. In the harmonic ellipsoidal quantum dot the center-of-mass motion separates from the relative motion eigenproblem. ${ }^{32}$ If the energy levels involved in the avoided crossing corresponded to different center-of-mass states, the level crossing would still be observed in spite of the same parity of the considered few-electron states. The appearance of the avoided level crossings for $N=2$ and 4 indicates that these levels are associated with the same (ground) state of the center of mass. In the two-electron system the energy gaps between the anticrossing energy levels $[\Delta E$, cf. Fig. 7(a)] for the same degree of anisotropy $\left(\omega_{y} / \omega_{x}\right)$ are about four times larger than in four-electron systems. The extent to which the anisotropy mixes the magic-angular-momenta states of circular dots is a distinctly decreasing function of the differences of their angular momenta $(N \hbar)$. The appearance of the avoided crossings in function of the anisotropy of the elliptical confinement in absence of a magnetic field has been discussed for an electron pair in Ref. 10.

The magnetic-field-induced ground-state transformations in the circular quantum dots are accompanied by cusps in the energy as a function of the magnetic field and stepwise changes of the angular momentum. Moreover, they appear along with discontinuous changes of the average size of the system, ${ }^{30}$ the electron-electron interaction energy, ${ }^{29}$ and abrupt transformations of the charge density. When the energy-level crossing is replaced by an avoided crossing (like in two and four elliptically deformed dots) the changes of physical quantities lose their sharp character and become continuous. As an illustration, a plot of the interaction energy in the two-electron system is presented in Fig. 8(a) for circular (solid line) and elliptical (dashed line) quantum dot. In the circular dot the interaction energy grows with magnetic field between the ground-state transformations which are ac-

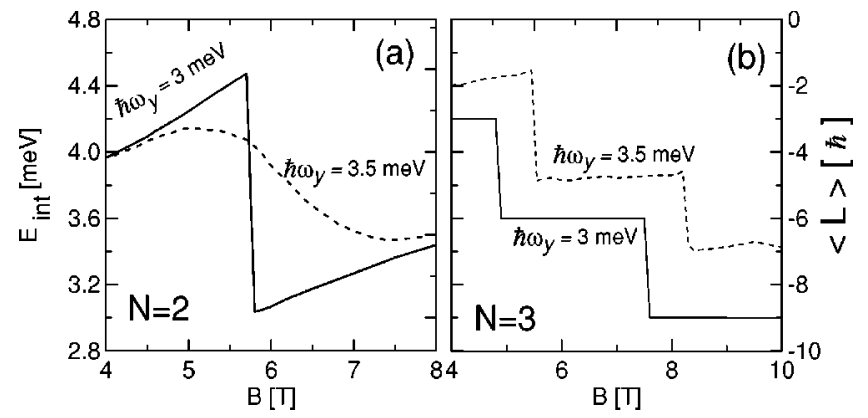

FIG. 8. Expectation value of the electron-electron interaction energy in the two-electron system (a) and of the angular momentum (b) in the three-electron circular ( $\hbar \omega_{x}=\hbar \omega_{y}=3 \mathrm{meV}$-solid lines) and elliptical ( $\hbar \omega_{x}=3, \hbar \omega_{y}=3.5 \mathrm{meV}$-dashed lines) quantum dot.

companied by a stepwise decrease of this quantity. The interaction energy for the two-electron elliptical dot presents a smooth dependence on the magnetic field [cf. dashed line in Fig. 8(a)]. In circular quantum dots the sharp breakdown of the MDD is related with a level crossing leading to a sudden increase of the electron-electron correlation ${ }^{30}$ and formation of a molecular configuration in the inner coordinates of the quantum system. ${ }^{2,3,29,30}$ In the presence of the avoided crossings the formation of the Wigner phase becomes a continuous process.

In the three-electron system confined in an elliptical dot the magnetic-field-induced level crossings are still present because, like in circular dots, the subsequent ground states possess opposite parities. Figure 9 shows the two lowestenergy levels as function of the magnetic field. One of the two lowest-energy levels corresponds to the odd-parity state (solid line) and the other to the even-parity state (dashed

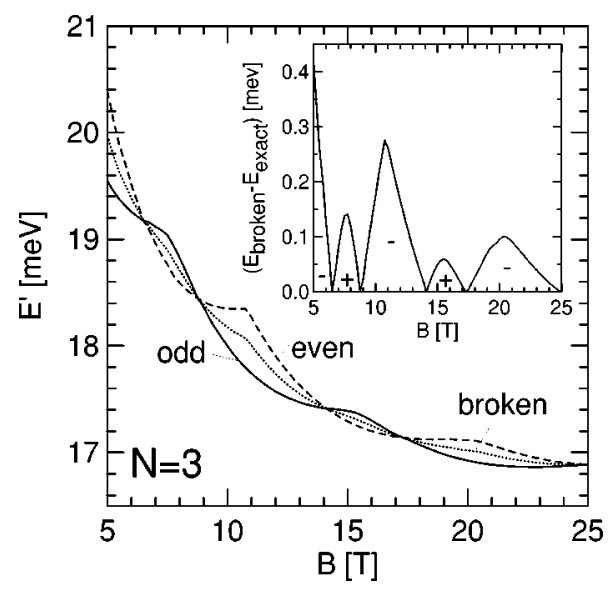

FIG. 9. Two lowest-energy levels of a three-electron elliptical $\operatorname{dot}\left(\hbar \omega_{x}=3 \mathrm{meV}, \hbar \omega_{y}=4 \mathrm{meV}\right)$ calculated with respect to the lowest Landau level. The energy level plotted with solid (dashed) line corresponds to the state of odd (even) parity. Dotted line shows the expectation value of the energy for the broken-symmetry state [cf. Sec. III C and Eq. (5)]. The inset presents half the energy spacing between the two energy levels, i.e., the energy overestimation by the expectation value of the Hamiltonian calculated for the broken-symmetry state (cf. Sec. III C). Signs " - " and "+" correspond to odd and even parity of the ground state, respectively. 


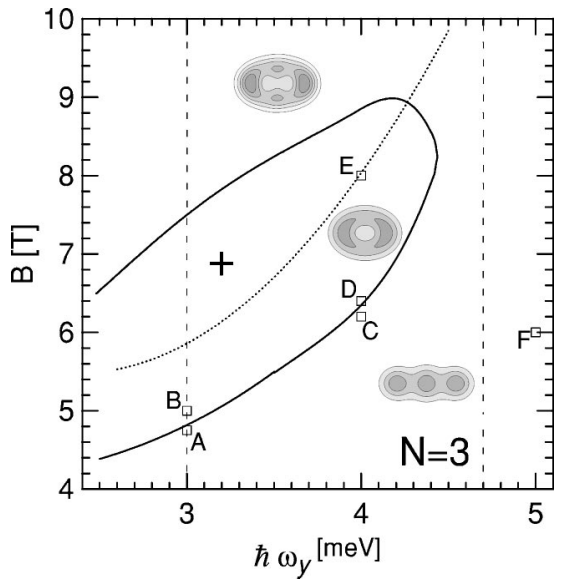

FIG. 10. Phase diagram for the parity of the three-electron spinpolarized ground state in an elliptical quantum dot for $\hbar \omega_{x}$ $=3 \mathrm{meV}$. The parity is even in the region marked by a plus sign bounded by the solid line and odd outside of it. The dotted line shows the position of the avoided crossing of the lowest two oddparity energy levels. The insets show schematically the qualitative picture of the charge density in the different phases. For the vertical dashed lines and the symbols, see text.

line). In the three-electron elliptical dot the changes of physical properties conserve their stepwise character as function of the magnetic field due to the parity transformations. Figure 8(b) shows the expectation value of the angular momentum for the three-electron system. Although, the angular momentum in an elliptical dot is not quantized, its expectation value presents discontinuous changes. For higher values of $\omega_{y}$ the angular momentum is quenched to zero as the confinement potential starts to resemble a quasi-one-dimensional wirelike dot.

Figure 10 shows the phase diagram for the parity of the spin-polarized three-electron system as a function of the strength of the $y$ confinement and the magnetic field for $\hbar \omega_{x}=3 \mathrm{meV}$. The ground state is of even parity in the central region bounded by the solid line and marked by the "+" sign. The left vertical dashed line corresponds to circular symmetry of the confinement potential. The changes in the charge density occurring at the crossing of the border between the odd and even phases along this line are illustrated in Fig. 11. The upper panels of Fig. 11 show the charge density (left panel) and the pair-correlation function (PCF) (right panel) corresponding to the MDD phase in the point marked by " $A$ " in Fig. 10. The lower panels correspond to the point marked by " $B$ " in Fig. 10. Results of Fig. 11 show that the MDD decay is accompanied by the formation of a hole in the charge-density center ${ }^{33}$ and a distinct growth of the electron separation in the inner coordinates presented at the PCF plots. Figure 12 displays the charge density and the PCF plots at the crossing of the border between the odd- and even-parity phases for elliptical dot with $\hbar \omega_{y}=4 \mathrm{meV}$ (cf. the points marked by " $C$ " and " $D$ " in Fig. 10). The charge density in the even-parity ground state has a hole in the center, similarly as for the even-parity state in the cylindrical dot (cf. Fig. 11). An increase of the electron-electron correlation appearing at the crossing of the borders between the phases

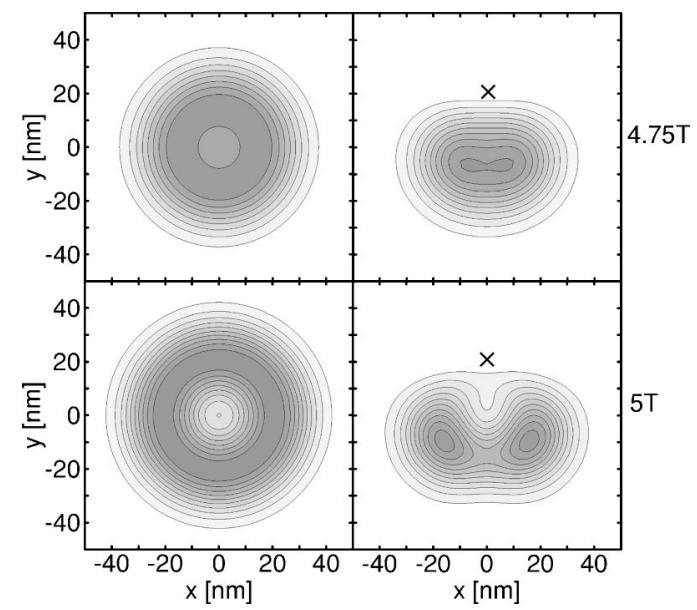

FIG. 11. Charge density (left panel) and PCF (right panel) plots for the three-electron system confined in a circular dot $\left(\hbar \omega_{x}\right.$ $=\hbar \omega_{y}=3 \mathrm{meV}$ ) in the MDD phase (upper panel cf. point marked by " $A$ " in Fig. 10) and in the ground state with $L=-6 \hbar$ appearing at higher magnetic field (lower panel cf. point marked by " $B$ " in Fig 10. The position of one of the electrons in the PCF plot marked by $\times$ is fixed at point $x=0, y=21 \mathrm{~nm}$.

also resembles a similar effect appearing in circular dots (cf. right panel of Fig. 12). The charge-density plot for the same phase at a higher magnetic field $(B=8 \mathrm{~T}$-point " $E$ " in Fig. 10) was presented in Fig. 4. A qualitative change in the charge density is observed at higher magnetic fields when the next phase border is crossed. In the odd phase above this border, the two local maxima of the charge density appear at the $y$ axis (cf. Fig. 4 for $B=12 \mathrm{~T}$ and $N=3$ ). At higher magnetic field the charge density tends to reproduce both the degenerate classical configurations (cf. discussion of Fig. 4).

Figure 10 shows that with increasing anisotropy of the confinement the even-parity phase is pushed to higher magnetic fields and finally for $\hbar \omega_{y}>4.4 \mathrm{meV}$ it is eliminated

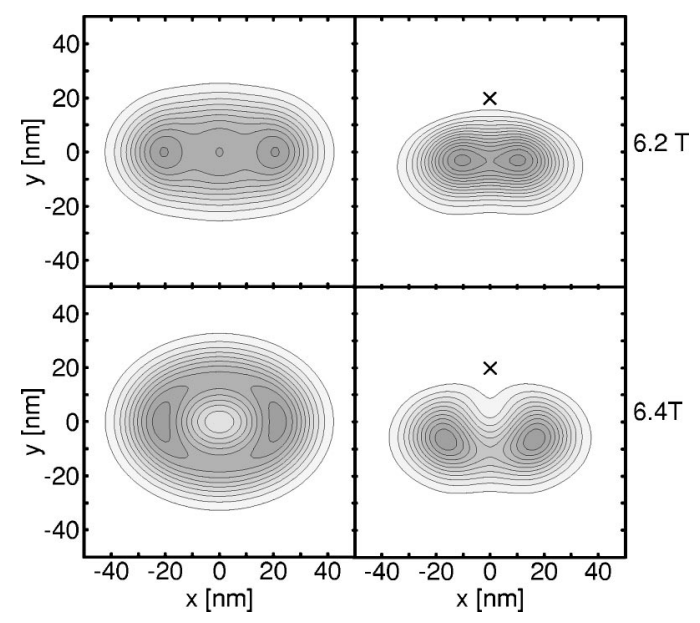

FIG. 12. Charge density (left panel) and PCF (right panel) plots for the three-electron system in an elliptical dot $\left(\hbar \omega_{x}=3 \mathrm{meV}\right.$, $\hbar \omega_{y}=4 \mathrm{meV}$ ). The upper (lower) panel corresponds to the odd(even-) parity state in the point marked by " $C$ " (" $D$ ") in Fig. 10. The position of one of the electrons in the PCF plot marked by $\times$ is fixed at point $x=0, y=20 \mathrm{~nm}$. 


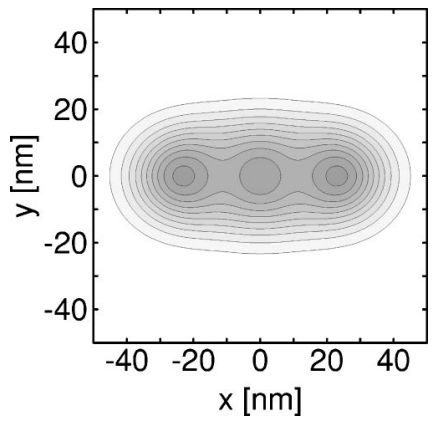

FIG. 13. Charge density for the three-electron system in an elliptical dot $\left(\hbar \omega_{x}=3 \mathrm{meV}, \hbar \omega_{y}=5 \mathrm{meV}\right)$ for $B=6 \mathrm{~T}$. The plot corresponds to the point marked by " $F$ " in Fig. 10 .

from the phase diagram. The charge-density plot presented in the upper panel of Fig. 12 for point " $C$ " in Fig. 10 shows that the odd-parity state forms a charge-density maximum in the center of the dot. The strong $y$ confinement prevents the formation of the even-parity state (the lower panel of Fig. 12) in which this central charge-density maximum is removed. The right vertical dashed line in Fig. 10 marks the $y$ confinement energy above which the zigzag structure ${ }^{18}$ of the classical three-electron system (cf. lowest panel of Fig. 4) is suppressed to the $x$ axis. Figure 13 shows the electron charge density plotted for $\hbar \omega_{y}=5 \mathrm{meV}$ and $B=6 \mathrm{~T}$ (the point marked by " $F$ " in Fig. 10). The observed three chargedensity maxima at higher magnetic field shrink and tend toward the single nondegenerate classical configuration. In the quasi-one-dimensional regime of strong $y$ confinement the convergence of the three-electron charge density to the classical limit is not accompanied by any level crossings.

\section{Broken-symmetry states}

The preceding results show that the quantum systems with classical degeneracy at high field contain all the classically degenerate configurations. As a consequence, the corresponding charge density does not resemble any single classical charge distribution. One has to break the symmetry of the external potential in order that the quantum charge density reproduces one of the degenerate classical configurations. Let us construct such broken-symmetry states $\psi_{b s}$ in form of a linear combination of the two lowest-energy fewelectron states ( $\chi_{1}$ and $\chi_{2}$, respectively),

$$
\psi_{b s}=\left(\chi_{1}+c \chi_{2}\right) / \sqrt{2},
$$

where $|c|^{2}=1$. Usually, the charge density of a state constructed in this way does not reproduce the symmetry of the confinement potential. We constructed such brokensymmetry states for the system of three electrons in an elliptical dot with $\hbar \omega_{x}=3 \mathrm{meV}$ and $\hbar \omega_{y}=4 \mathrm{meV}$ (cf. Fig. 9). We have found that the broken-symmetry charge density exhibits three maxima. For a properly chosen phase of the coefficient $c$ in Eq. (5) the positions of these maxima coincide with the position of the electrons in one of the degenerate classical configurations (cf. the lowest panel of Fig. 4). Figure 14 shows the charge density of the broken-symmetry states for different values of the magnetic field. Plots in Figs.

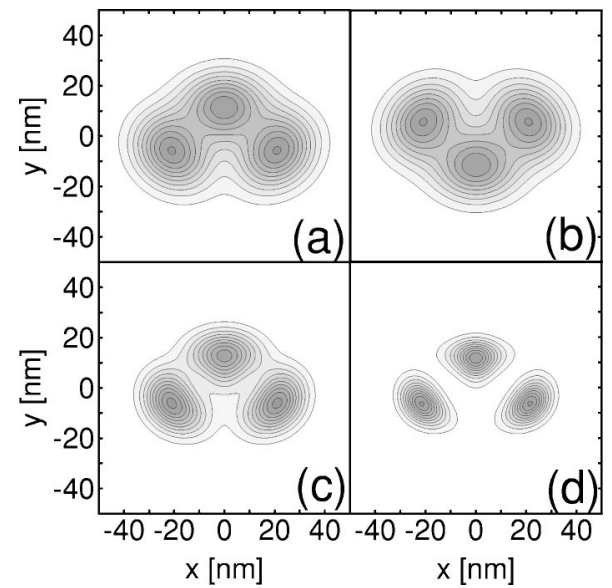

FIG. 14. Charge density of the broken-symmetry three-electron states [cf. Eq. (5)] in an elliptical dot $\left(\hbar \omega_{x}=3 \mathrm{meV}, \hbar \omega_{y}\right.$ $=4 \mathrm{meV}$ ) for $B=6.3 \mathrm{~T}(\mathrm{a}, \mathrm{b}), 14.125 \mathrm{~T}(\mathrm{c})$, and $25 \mathrm{~T}(\mathrm{~d})$. For the chosen fields the plots correspond to exact ground states.

14(a) and 14(b) correspond to "black" and "white" degenerate classical configurations depicted in the lowest panel of Fig. 4. Plots 14(a) and 14(b) have been obtained with opposite sings of $c$ in formula (5), so they correspond to orthogonal wave functions. Figures 14(a), 14(c), and 14(d) show that as the magnetic field grows the charge density of the brokensymmetry state converges to one of the degenerate classical configurations of point charges.

The expectation value of the energy calculated for the broken-symmetry state [Eq. (5)], independently of the phase of $c$, is equal to the arithmetic average of the two lowestenergy levels. It means that this expectation value overestimates the exact ground-state energy by half of the energy spacing between the two lowest levels (cf. inset of Fig. 9). For the magnetic fields corresponding to degenerate $\chi_{1}$ and $\chi_{2}$ states [cf. Eq. (5)], i.e., to the energy-level crossings presented in Fig. 9, the broken-symmetry states (5) are exact ground eigenstates of the Hamiltonian. The magnetic fields chosen in Fig. 14 correspond to these level crossings. Therefore, the charge densities presented in this figure correspond to the exact ground-state solutions of the Schrödinger equation. Conversely, for an arbitrary value of the magnetic field the exact ground-state wave function can be constructed from a superposition of the wave functions of two brokensymmetry states with semiclassical electron localization [cf. Figs. 14(a) and 14(b)].

\section{SQUARE QUANTUM DOT}

In order to verify the conclusions concerning the highmagnetic-field evolution of the charge density in elliptical dots we performed a study of Wigner crystallization in quantum dots with square and triangular confinement potentials. For the square quantum dot we used a smooth confinement potential with a square profile,

$$
V(x, y)=\frac{1}{2} m^{*} \omega^{2}\left(x^{2}+y^{2}\right)[1+\cos (4 \phi) / 5],
$$

where $\phi$ is the angle between the position vector $(x, y)$ and the $x$ axis. We take $\hbar \omega=3 \mathrm{meV}$. The potential is illustrated 


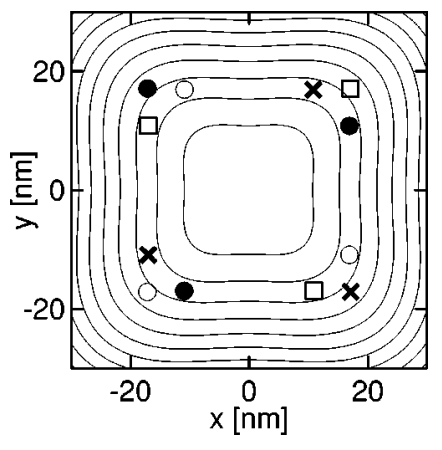

FIG. 15. Equipotential lines for the square confinement potential (6). The different types of symbols mark the position of the electrons in one of the four energy-equivalent configurations for the three-electron system.

in Fig. 15 along with the degenerate classical lowest-energy configurations of three electrons. On the other hand for two electrons there are only two equivalent configurations in which the electrons reside in the opposite corners of the square, ${ }^{34}$ and for $N=4$ the classical system is nondegenerate with electrons occupying all the corners.

The MCI calculations for potential (6) have been performed with 12 centers placed on the circumference of a square with equal spacings along its sides. The size of the square was optimized variationally. The obtained charge density is presented in Fig. 16. In the system of four electrons the charge density becomes distinctly separated into four single-electron islands. For $N=2$ and 3 the formation of the charge maxima at the corners of the square appears with a pronounced delay in magnetic-field strength with respect to the four-electron system.

The PCF plot presented in Fig. 17 gives an additional insight into the electron distribution in the square quantum

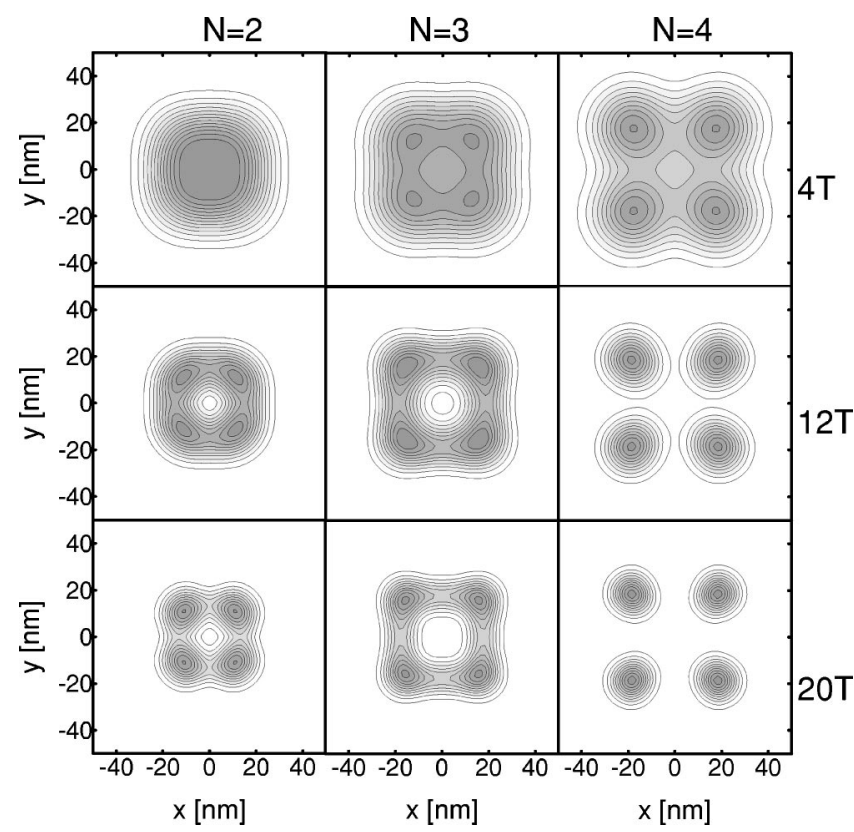

FIG. 16. Charge densities of two-, three-, and four-electron systems in the square quantum dot for various values of the magnetic field.

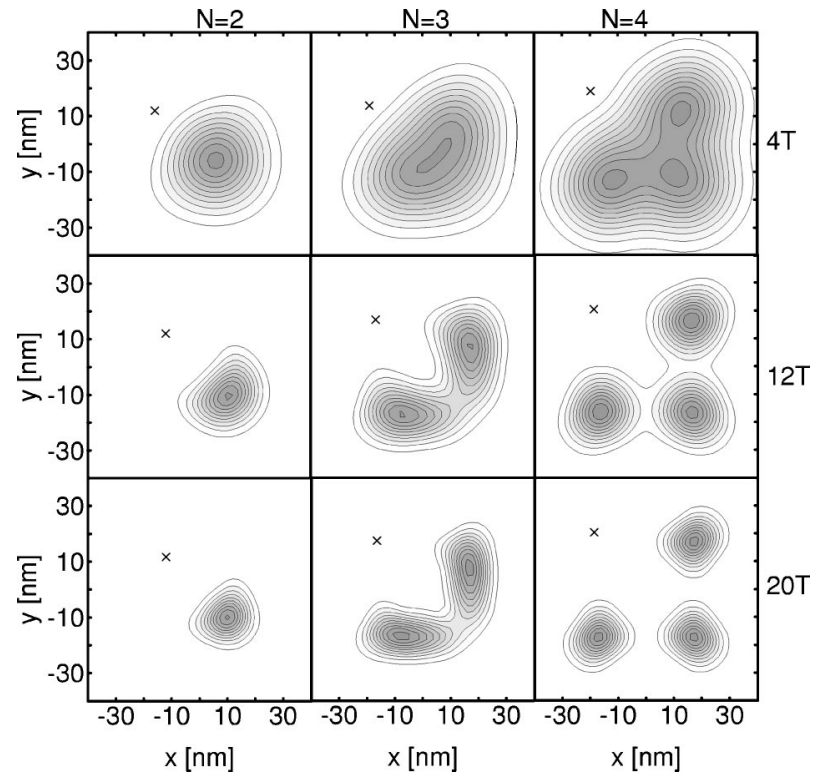

FIG. 17. Pair-correlation function for the two-, three-, and fourelectron system in the square quantum dot. The cross marks the position of one of the electrons $[(-12,12),(-17,17),(-20,20)$ for $N=2,3$, and 4, respectively (in nanometers)].

dot when one of the electrons is fixed (cross in Fig. 17) along one of the diagonals. At high magnetic field the plots for $N$ $=2$ and 4 show that the other electrons become localized semiclassically at the corners of the square. On the other hand the two remaining electrons in the three-electron system are smeared out over the two opposite sides of the square and their localization is weaker than in the two- and four-electron systems. This weaker localization is related to the degeneracy of the classical three-electron system and to the fact that the electrons in the degenerate classical configurations occupy nearby positions.

Table II shows that the present MCI approach gives a decent convergence of the energy estimates even for the three-electron system whose localization in the square quantum dot is rather vicious.

The gathering of the electron density at the corners of the square dot that we observe at high magnetic field is in qualitative agreement with previous exact ${ }^{9}$ and density-functional-theory ${ }^{11,12}$ results for large quantum dots in the absence of magnetic field.

The symmetry of the electron states in square quantum dots is higher than in elliptical dots. ${ }^{35}$ In the symmetric gauge the Hamiltonian eigenstates are also eigenstates of the

TABLE II. Convergence of the total energy (in meV) for the three-electron system in the square quantum dot (6) as function of the number of centers used in the wave function (3).

\begin{tabular}{lcccc}
\hline \hline$B(\mathrm{~T})$ & \multicolumn{5}{c}{$\mathrm{M}$} \\
\hline 4 & 4 & 8 & 12 & 16 \\
12 & 28.51 & 28.08 & 27.99 & 27.98 \\
20 & 48.87 & 46.38 & 46.27 & 46.26 \\
\hline \hline
\end{tabular}




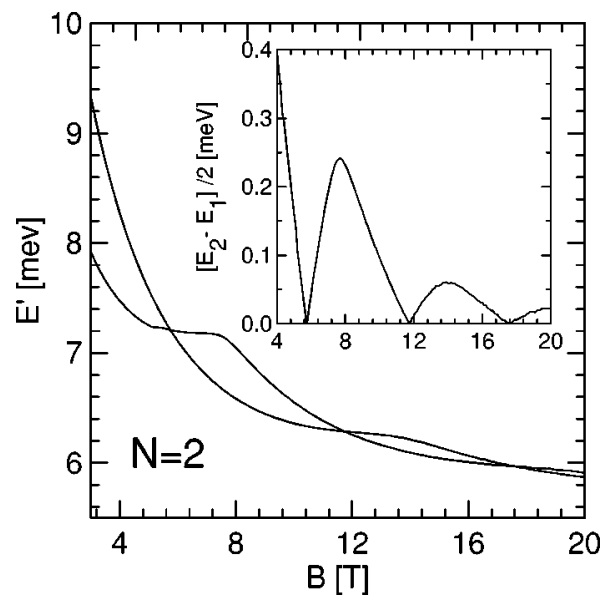

FIG. 18. Two lowest-energy levels of the two-electron square quantum dot calculated with respect to the lowest Landau level as functions of the magnetic field. Both energy levels are of odd parity. The inset shows half of the energy spacing between the two lowestenergy levels.

$\pi / 2$ rotation operator corresponding to eigenvalues \pm 1 (even-parity states) and $\pm i$ (odd-parity states). Since we are using the Landau gauge, in which the Hamiltonian does not commute with the $\pi / 2$ rotation operator, we cannot discuss these symmetries properly. ${ }^{36}$ However, we have found that the ground state of two electrons for the MDD and the other spin-polarized ground states at high magnetic fields is always of odd-parity-like in circular and elliptical quantum dots. Figure 18 shows the two lowest-energy levels of twoelectrons in a square quantum dot (the lowest excited state is also of odd parity). Contrary to the case of elliptical dots the crossings between the odd-parity energy levels are not avoided. Thus we can conclude that the interchanging twoelectron energy levels presented in Fig. 18 correspond to orthogonal eigenstates with eigenvalues $\pm i$ of the $\pi / 2$ rotation operator.

For three electrons the oscillations of the ground-state parity with magnetic field are observed like in circular and elliptically deformed dots (cf. Fig. 9). The two lowest-energy levels for $N=3$ are presented in Fig. 19.

The two- and three-electron systems in square quantum dots are similar to the three-electron system in an elliptically deformed dot (with comparable confinement energies in the $x$ and $y$ directions) in three points. First, all these systems exhibit classical degeneracy. Second, their energy levels exhibit crossings as function of the magnetic field (cf. Figs. 13, 18, and 19). Third, it is possible to extract a single semiclassical broken-symmetry charge distribution as a linear combination of the two crossing lowest-energy levels.

Figure 20 shows the charge density of a superposition of the two lowest-energy states of two- and three-electron square dot calculated using Eq. (5). The other semiclassical configuration of the two-electron system corresponding to electrons gathering at the other diagonal of the square can be obtained by changing the sign of $c$ in Eq. (5). The threeelectron charge density plotted in Figs. 20(c) and 20(d) corresponds to the classical charge density marked by squares in Fig. 15. The three other equivalent configurations can be

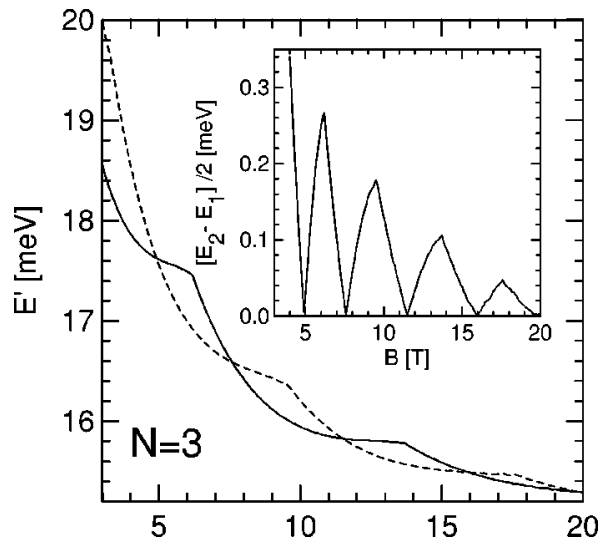

FIG. 19. Two lowest-energy levels of the three-electron square quantum dot calculated with respect to the lowest Landau level. The odd- (even-) parity energy level is plotted with solid (dashed) line. The inset presents the half of the energy spacing between the two lowest-energy levels.

obtained by rotating the coefficient $c$ by $\pi / 2$ in the Gauss plane. It is interesting to note that a linear combination of the two lowest-energy states can yield all four semiclassical charge distributions. On the other hand any pair of the four broken-symmetry states is sufficient to reconstruct the exact ground state. The broken-symmetry states corresponding to the classical configurations marked by squares and open dots (full dots and crosses) in Fig. 15 are mutually orthogonal. With increasing magnetic field the charge maxima presented in Fig. 20 shrink to the classical point-charge distributions.

The energy overestimate of the broken-symmetry state (half of the energy spacing between the lowest levels) is presented in the insets of Figs. 18 and 19, respectively. We notice that the envelope of the oscillation of the energy overestimate is a decreasing function of the magnetic field. Similar decreasing tendency can be noticed for the three-electron system in an elliptical dot (cf. inset of Fig. 9), but in that case the parity of the ground state has a visible influence on the height of the local maxima of the energy overestimate due to the shrinkage of the stability region of the even-parity phase with growing $y$-confinement energy (cf. Fig. 10).

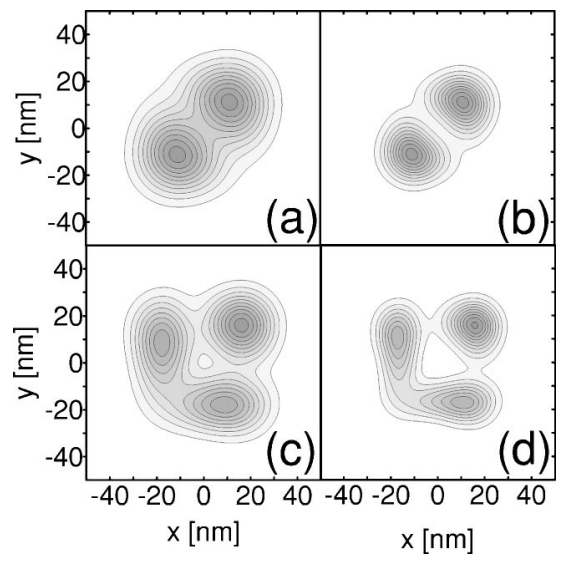

FIG. 20. Charge density of the broken-symmetry solutions [cf. Eq. (5)] of the two- (a, b) and three-electron square quantum dot, for $B=5 \mathrm{~T}(\mathrm{a}), 12 \mathrm{~T}(\mathrm{~b}), 8 \mathrm{~T}(\mathrm{c})$, and $16 \mathrm{~T}$. 


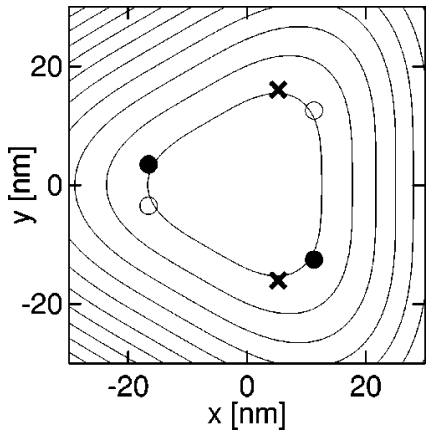

FIG. 21. Equipotential lines for the triangular confinement potential (7) with $\hbar \omega=3 \mathrm{meV}$. Different symbols show the three degenerate classical lowest-energy configurations for the two-electron system.

The four-electron spin-polarized ground state in a square dot is of even parity (like in circular and elliptical dots) and no level crossings as function of the magnetic field are observed. In this respect the four-electron system confined in a square quantum dot is similar to the two- and four-electron systems in elliptical dots as well as to three-electrons in strongly deformed wirelike quantum dots (cf. discussion of Fig. 10). Another feature common to almost all these systems is that their classical counterpart is nondegenerate. The only exception is the four-electron system for elliptical dots with $\omega_{y} / \omega_{x}$ ratio corresponding to the zigzag classical configuration (cf. Fig. 6). We did not obtain level crossing for this system, although for certain magnetic fields the excited oddparity state can closely approach the even ground state.

\section{TRIANGULAR QUANTUM DOT}

The confinement potential for a triangular dot is taken as ${ }^{7}$

$$
V(x, y)=\frac{1}{2} m^{*} \omega^{2}\left(x^{2}+y^{2}\right)[1+2 \cos (3 \phi) / 7],
$$

with $\hbar \omega=3 \mathrm{meV}$. Classical three- and four-electron systems in this potential are nondegenerate; the electrons occupy all the corners of the triangle, and one of the electrons in the four-electron system resides in the center of the triangle. ${ }^{34}$ On the other hand the classical two-electron system is threefold degenerate. The profile of the potential and the positions of electrons in the degenerate two-electron classical configurations are presented in Fig. 21.

The calculations were performed with 12 centers situated at the circumference of an equilateral triangle with equal spacing along its sides. For four electrons an additional 13th center was introduced in the center of the triangle. As in the preceding calculations, the size of the triangle was optimized variationally. The obtained charge density is plotted in Fig. 22. In systems of three and four electrons the magnetic field induces the formation of single-electron islands around the positions of classical electrons in the nondegenerate lowestenergy configurations. The clear localization of electrons for $N=3$ and 4 resembles the one for four-electrons in the square quantum dot (cf. Fig. 16) as well as the plots for $N$ $=2$ and 4 in the anisotropic confinement potential presented in Fig. 4. On the other hand for $N=2$ the formation of the

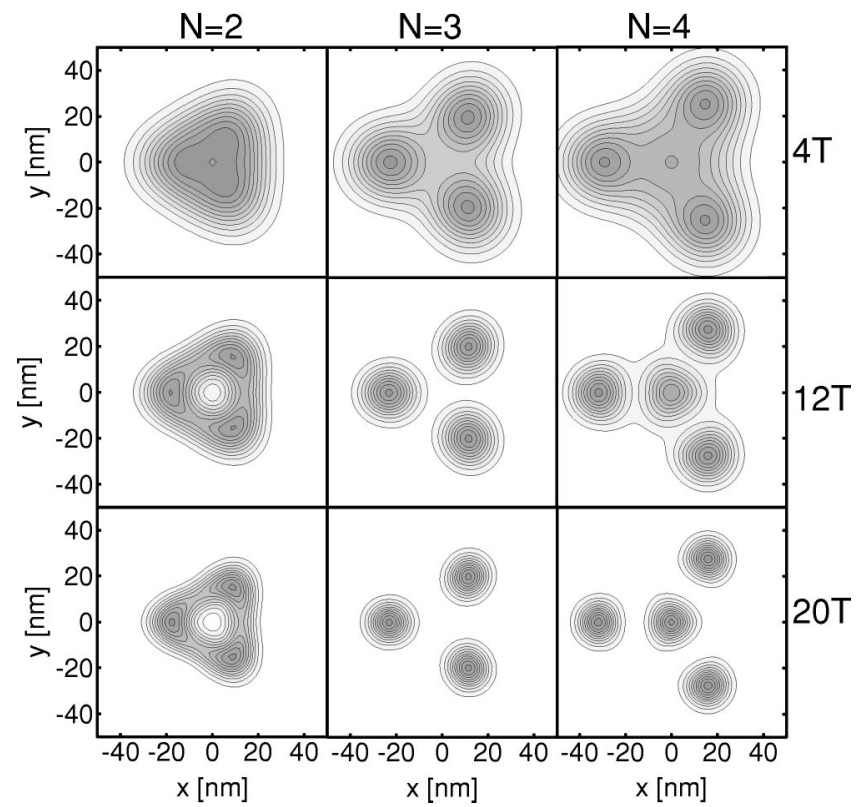

FIG. 22. Charge densities of two-, three-, and four-electron systems in a triangular quantum dot (7) with $\hbar \omega=3 \mathrm{meV}$ for various values of the magnetic field.

charge maxima at the corners of the triangle appears with a visible delay and the localization of electrons along the sides is observed like in the three-electron system in the square quantum dot (cf. Fig. 16).

The three- and four-electron systems in the triangular dot, for which their classical lowest-energy configuration is nondegenerate, do not exhibit any level crossings as function of the magnetic field, but the two-electron system (classically degenerate) does. The crossing lowest-energy levels are presented in Fig. 23. The electron systems in the triangular confinement potential do not possess a definite parity. However, (in the symmetric gauge) the Hamiltonian eigenfunctions should also be eigenstates of the $2 \pi / 3$ rotation operator cor-

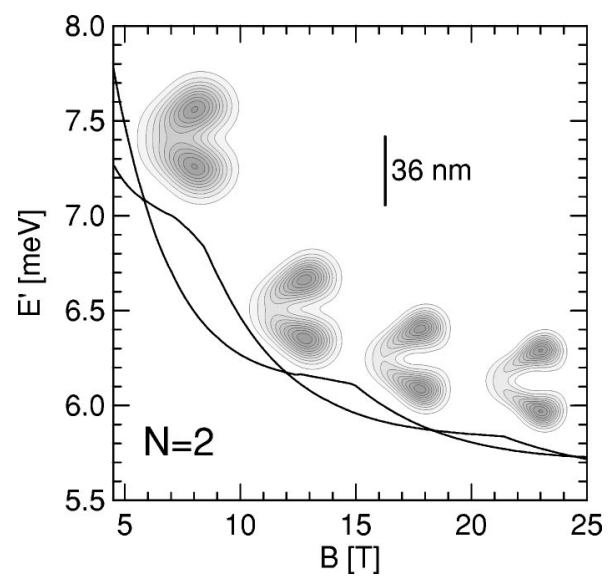

FIG. 23. Two lowest-energy levels of a two-electron triangular quantum dot calculated with respect to the lowest Landau level. The insets present the charge density of the broken-symmetry solutions obtained for the subsequent energy-level crossings appearing at magnetic fields $5.9,12,18.3$, and $24.4 \mathrm{~T}$, respectively. The bar in the inset shows the length scale for the charge-density plots. 


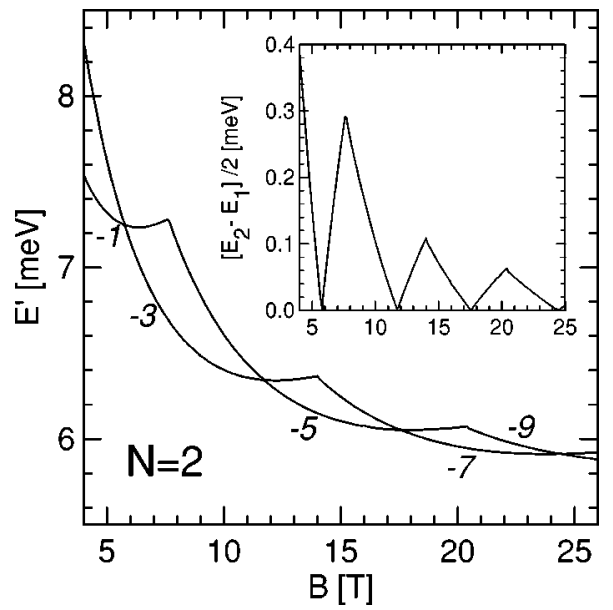

FIG. 24. Two lowest-energy levels of the two-electron system in circular dot with $\hbar \omega=3 \mathrm{meV}$ calculated with respect to the lowest Landau level. The numbers $(-1,-3,-5,-7$, and -9$)$ give the angular momenta of the two lowest-energy states (in $\hbar$ units).

responding to eigenvalues equal to three complex cubic roots of 1. It may be expected that each level of the crossing pairs presented in Fig. 23 corresponds to a different eigenvalue. Similarly as in the dots studied in the preceding part of the paper the energy spacings between the two lowest levels exhibit oscillations decreasing with the external field. Formula (5) still successfully produces the semiclassical chargedensity distributions. The insets of Fig. 23 show the plots of the broken-symmetry solutions drawn for the magnetic fields corresponding to the level crossings. The presented charge densities correspond to the classical configuration marked by crosses in Fig. 21. We have verified that the other two semiclassical distributions can be obtained by rotation of $c$ in Eq. (5) by $2 \pi / 3$ in the Gauss plane.

\section{EXACT BROKEN-SYMMETRY STATES FOR CIRCULAR DOTS}

From the present findings for anisotropic dots we may wonder whether it is also possible to obtain the classical configurations for circular dots. The classical electron systems in circular dots are infinitely degenerate with respect to rotation over an arbitrary angle. From the point of view of the preceding discussion, the cylindrical symmetry of the exact charge density can be considered as a superposition of all classically degenerate configurations. Like most of the classically degenerate systems discussed in this paper, the electron systems in circular quantum dots exhibit level crossings as function of the magnetic field.

The two lowest-energy levels of the two-electron circular dot are displayed in Fig. 24. For an arbitrary magnetic field the ground and the first excited states correspond to adjacent magic angular momenta (given by numbers close to the curves in Fig. 24). The inset shows half of the energy spacing between the lowest levels. The superposition of the two lowest-energy states calculated according to Eq. (5) give the broken-symmetry semiclassical distributions which are displayed in Fig. 25. The magnetic fields chosen for Fig. 25 are

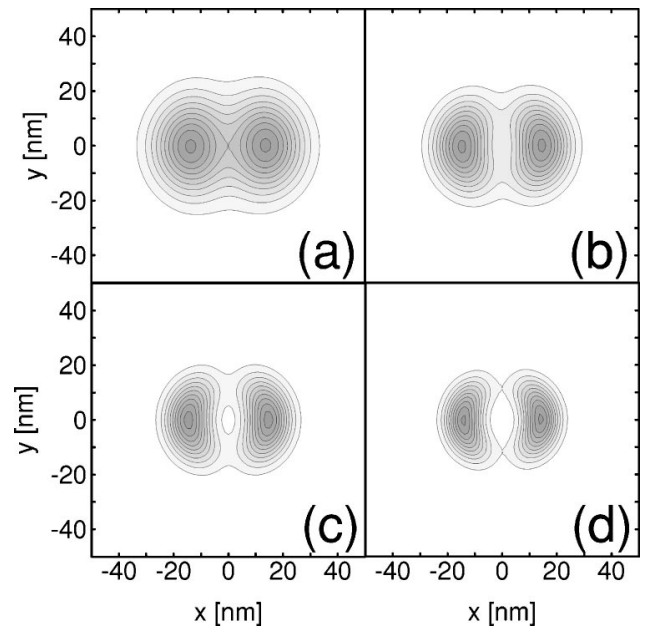

FIG. 25. Broken-symmetry charge densities of the superposition of two lowest-energy states for $N=2$ for a circular dot. Plots (a-d) correspond to magnetic fields $6,11.5,17.5$, and $25 \mathrm{~T}$, respectively.

close to the level crossings presented in Fig. 24. At the level crossings the broken-symmetry states are exact ground states. The corresponding charge densities shrink to pointlike density distributions with growing magnetic field. The modification of the phase of $c$ in Eq. (5) results in a rotation of the broken-symmetry Wigner molecules, which can be pinned at an arbitrary angle for a properly chosen phase. ${ }^{37}$ It is a striking feature of the quantum superposition [Eq. (5)] that for an arbitrary magnetic field the exact ground state with circularly symmetric charge density can be reconstructed from the wave functions of two broken-symmetry Wigner molecules pinned at any two different angles [cf. also the discussion in connection with Figs. 20(c) and 20(d)].

The energy levels of the three- and four-electron system (cf. Figs. 26 and 27) exhibit the same qualitative behavior as for $N=2$. The envelope of the lowest-energy-level separation presented for $N=2,3$, and 4 electrons in the insets of Figs. 24, 26 and 27 exhibits very similar dependence on the magnetic field, however "the frequency" of these oscillations grows fast with the number of electrons. The brokensymmetry charge densities in the neighborhood of the

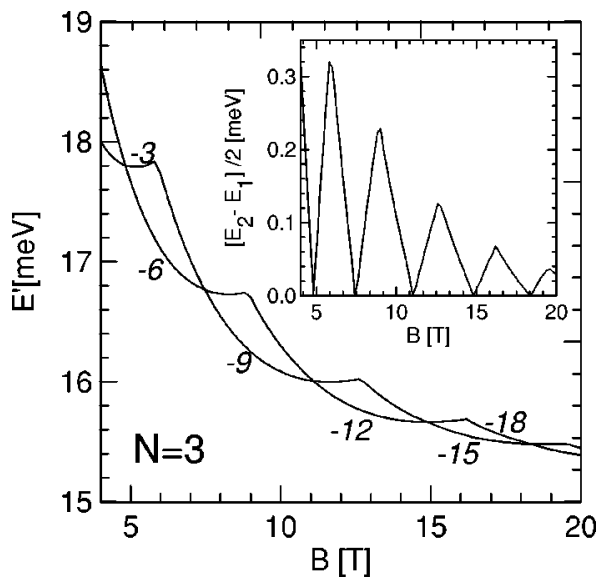

FIG. 26. Same as Fig. 24 but for $N=3$. 


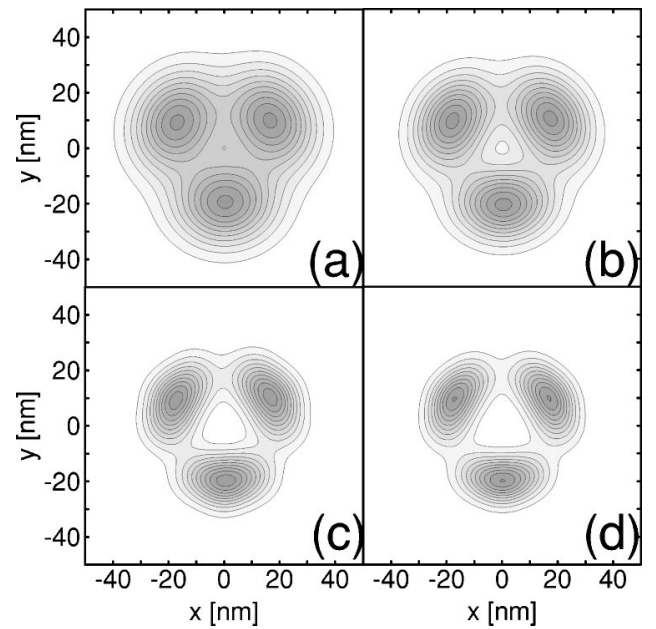

FIG. 27. Broken-symmetry charge densities for $N=3$ for a circular dot. Plots (a-d) correspond to magnetic fields 4.9, 7.5, 15, and $18.4 \mathrm{~T}$, respectively.

ground-state level crossings for $N=3$ and 4 are displayed in Figs. 27 and 29. The charge density presented in Fig. 27(a) has been obtained as a superposition of states with angular momenta $-3 \hbar$ and $-6 \hbar$. The charge densities of these states in the neighborhood of their energy-level crossings were shown in Fig. 11.

The present broken-symmetry charge densities, which are constructed from the superposition of the exact diagonalization solutions, are very similar to charge densities obtained by the unrestricted Hartree-Fock method, e.g., compare Fig. 27 with Fig. 13 of Ref. 38.

The present finding provides an insight into the problem of the magic angular momenta., ${ }^{2,20-22} \mathrm{~A}$ linear combination of any pair of states with different angular momenta produces a broken-symmetry charge density. The exceptional feature of the states with adjacent magic angular momenta is that their superposition reproduces the semiclassical charge density, which at the infinite magnetic-field limit tends to one of the degenerate classical point-charge distributions. It is obvious that any charge distribution, including the semiclassical one, can be reproduced by a superposition of angular-

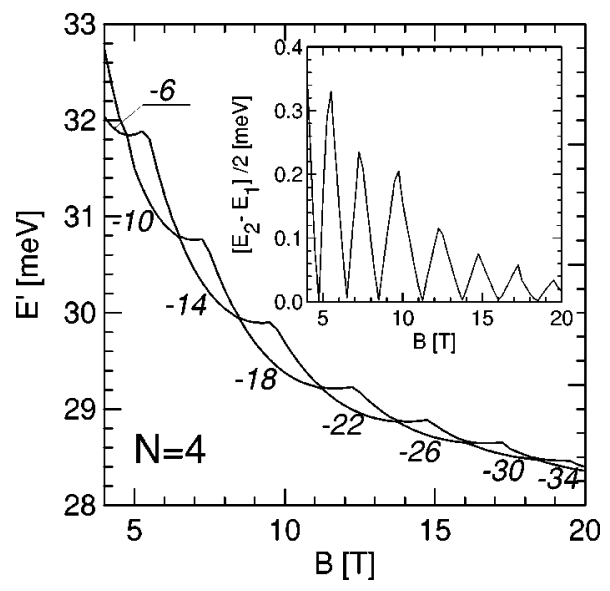

FIG. 28. Same as Fig. 24 but for $N=4$.

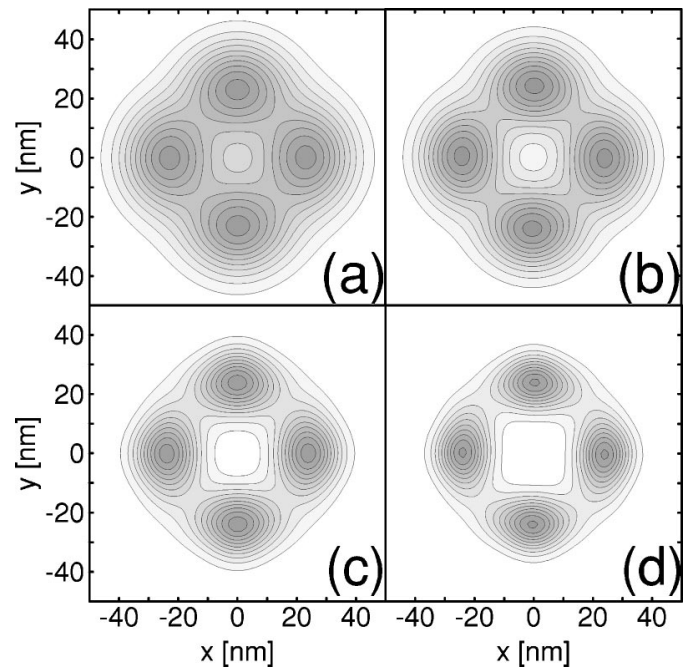

FIG. 29. Broken-symmetry charge densities for $N=4$ for a circular dot. Plots (a-d) correspond to magnetic fields 4.75, 6.5, 11.5, and $16 \mathrm{~T}$, respectively.

momentum eigenstates, since they form a complete basis. However, a superposition of more than two states would never give an exact value of the ground-state energy, since the ground state in spin-polarized circular dots is at most twofold degenerate. The subsequent magic angular momenta correspond to states which exhibit ground-state level crossing (each of the crossing levels corresponds to the ground state at its side of the level crossing). The level crossings allow the semiclassical laboratory-frame charge distribution to appear as a realizable feature of the exact ground state at the magnetic field corresponding to the crossing. We have to note here that this conclusion has been reached for systems containing a small number of electrons and we cannot exclude a different behavior for larger $N$.

Mean-field approaches predict spontaneous breaking ${ }^{3}$ of the symmetry of the confinement potential symmetry of the electron wave function after the MDD decay. Although, this effect is a notorious artifact, ${ }^{3}$ it is generally believed that there is some deeper physics behind it. The exact solutions show a rapid increase of the electron-electron correlation after the MDD breakdown. This increase appears in the inner coordinates and can be observed in the PCF plots (cf. Figs. 11, 12, and Ref. 30). Since the mean-field theories cannot give a complete description of the inner-coordinate space, they tend to account for the electron-electron correlation in the external (laboratory) frame of reference, which results in the symmetry breaking.

The energy overestimates obtained with the brokensymmetry solutions exhibit oscillations with amplitude decreasing with the magnetic field. The precision of the ground-state energy estimates obtained by the HF wave function with semiclassical localization ${ }^{24}$ also possesses an oscillatory dependence on the magnetic field with minima at the magnetic field corresponding to the exact ground-state transformations. Contrary to the broken-symmetry solutions obtained in the present paper at the exact-diagonalization level the HF energy overestimates take on nonzero values at their 
minima. ${ }^{24}$ The present paper shows that the fact that the HF broken-symmetry solutions overestimate the exact groundstate energy at the fields inducing its transformations is not due to their broken symmetry, but to their mean-field character. The broken-symmetry states constructed from the degenerate exact solutions presented in this paper contain a complete description of the inner-coordinate space. Due to this fact they provide the exact ground-state energy for finite values of the magnetic fields corresponding to the groundstate transformations. The HF broken-symmetry solutions account for the separation of the electron charges but the rest of the correlation effects, most probably the reaction of an electron on the actual positions of the electrons inside the other charge puddles, is missed. This missing part of the correlation is squeezed to zero in the infinite magnetic field for which the charge puddles shrink to point-charge distributions. ${ }^{24}$

\section{SUMMARY AND CONCLUSIONS}

We presented a detailed study of the magnetic-fieldinduced Wigner crystallization of the two-, three-, and fourelectron spin-polarized systems in quantum dots. The obtained results, although limited to small numbers of electrons, cover several symmetries of the confinement potential. We studied quantum dots of elliptical, square, triangular, and circular symmetry. In the present study, we developed a configuration-interaction scheme which was based on single-electron wave functions expanded in a multicenter basis. The method, verified for the well-known case of an isotropic harmonic-oscillator potential, can be applied to the case of any smooth confinement potential with arbitrary symmetry. The arbitrariness in the choice of centers in basis (3) allows us to achieve a high accuracy and flexibility of the present MCI method accompanied with its relatively simple applicability to low-symmetry nanostructures.

We have studied the parity transformations in the spinpolarized electron systems confined in elliptical quantum dots and found anticrossings between the energy levels of the same spatial symmetry. The experimental identification ${ }^{39,40}$ of the magnetic-field-induced ground-state transformations for the $N$-electron system in a cylindrical quantum dot is based on detection of cusps of the chemical potential, i.e., the difference of the ground-state energy of the $N$ and $N$ -1 electron system. Based on the present results we expect that the ellipsoidal deformation of the quantum dot potential results in a smoothening of the cusps of the charging lines ${ }^{41}$ corresponding to the ground-state transformations between states of the same spin and parity symmetry. On the other hand, cusps related to spin or parity transformations should remain sharp.

Quantum Wigner molecules in anisotropic quantum dots are related with the lowest-energy configurations of their classical counterparts. At high magnetic fields the quantum charge density tends to simultaneously reproduce all the degenerate lowest-energy classical configurations. Classical degeneracy occurs when the lowest-energy configuration is of a different symmetry than the confinement potential. We found that the quantum charge density is a superposition of all these degenerate classical configurations. Consequently, the quantum-mechanical charge-density reproduces the symmetry of the confinement potential. We conclude that the observation of Wigner crystallization through its charge-density distribution will be facilitated in low-symmetry quantum dots for which the symmetry of the classical configuration conforms with the symmetry of the external potential, i.e., in systems which do not exhibit classical degeneracy.

Moreover, we have found a relation between the occurrence of the magnetic-field-induced level crossings and classical degeneracy. None of the studied quantum systems with nondegenerate classical counterpart exhibit such crossings. The formation of Wigner phase in these systems is a continuous process. For majority of the studied quantum systems with degenerate classical counterparts these crossings are observed. The only exception is the four-electron system for elliptical dots for which the classical configuration has a zigzag form. We have shown that in the presence of the crossings a superposition of the two lowest-energy states produces a broken-symmetry state whose charge density reproduces one of the degenerate classical configurations. These brokensymmetry states are exact ground states for the magnetic fields for which the crossings appear and for which the ground state is twofold degenerate. The ground-state degeneracy allows the semiclassical broken-symmetry charge distribution to be a realizable property of the quantum system. This conclusion holds also for circular dots. The superposition of the adjacent magic-angular-momenta states allows the inner symmetry of the quantum system to appear in the laboratory frame of reference.

\section{ACKNOWLEDGMENTS}

This paper has been partly supported by the Polish Ministry of Scientific Research and Information Technology in the framework of the solicited grant PBZ-MIN-008/P03/ 2003, the Flemish Science Foundation (FWO-Vl), the Concerted Action program (IUAP), and the University of Antwerpen (VIS and GOA). One of us (B.S.) was supported by the Foundation for Polish Science (FNP).
${ }^{1}$ L. Jacak, P. Hawrylak, and A. Wójs, Quantum Dots (Springer, Berlin, 1998).

${ }^{2}$ P.A. Maksym, H. Immamura, G.P. Mallon, and H. Aoki, J. Phys.: Condens. Matter 12, R299 (2000).

${ }^{3}$ S.M. Reimann and M. Manninen, Rev. Mod. Phys. 74, 1283 (2002).
${ }^{4}$ A. Matulis and F.M. Peeters, Solid State Commun. 117, 655 (2001).

${ }^{5}$ V.M. Bedanov and F.M. Peeters, Phys. Rev. B 49, 2667 (1994).

${ }^{6}$ E.E. Vdovin, A. Levin, A. Patanè, L. Eaves, P.C. Main, N.Yu. Khanin, Yu.V. Dubrovkii, M. Henini, and G. Hill, Science 290, 122 (2000). 
${ }^{7}$ T. Ezaki, N. Mori, and C. Hamaguchi, Phys. Rev. B 56, 6428 (1997).

${ }^{8}$ M. Manninen, M. Koskinen, S.M. Reimann, and B. Mottelson, Eur. Phys. J. D 16, 381 (2001).

${ }^{9}$ C.E. Creffield, W. Häusler, J.H. Jefferson, and S. Sarkar, Phys. Rev. B 59, 10719 (1999).

${ }^{10}$ P.S. Drouvelis, P. Schmelcher, and F.K. Diakonos, Europhys. Lett. 64, 232 (2003).

${ }^{11}$ E. Räsänen, H. Saarikoski, M.J. Puska, and R.M. Nieminen, Phys. Rev. B 67, 035326 (2003).

${ }^{12}$ S. Akbar and I.-L. Lee, Phys. Rev. B 63, 165301 (2001).

${ }^{13}$ D.G. Austing, S. Sasaki, S. Tarucha, S.M. Reimann, M. Koskinen, and M. Manninen, Phys. Rev. B 60, 11514 (1999).

${ }^{14}$ E. Räsänen, H. Saarikoski, V.N. Stavrou, A. Harju, M.J. Puska, and R.M. Nieminen, Phys. Rev. B 67, 235307 (2003).

${ }^{15}$ A. Natori, Y. Sugimoto, and M. Fujito, Jpn. J. Appl. Phys., Part 1 36, 3960 (1997).

${ }^{16}$ B. Reusch and R. Egger, Europhys. Lett. 64, 84 (2003).

${ }^{17}$ A.D. Güçlü, J.-S. Wang, and H. Guo, Phys. Rev. B 68, 035304 (2003).

${ }^{18}$ L. Cândido, J.-P. Rino, N. Studart, and F.M. Peeters, J. Phys.: Condens. Matter 10, 11627 (1998).

${ }^{19}$ A.H. MacDonald, S.R.E. Yang, and M.D. Johnson, Aust. J. Phys. 46, 345 (1993).

${ }^{20}$ S.M. Girvin and T. Jach, Phys. Rev. B 28, 4506 (1983).

${ }^{21}$ P.A. Maksym, Phys. Rev. B 53, 10871 (1996).

${ }^{22}$ C.G. Bao, W.Y. Ruan, and Y.Y. Liu, Phys. Rev. B 53, 10820 (1996)

${ }^{23}$ C. Yannouleas and U. Landman, Phys. Rev. B 66, 115315 (2002); 68, 035326 (2003)

${ }^{24}$ B. Szafran, S. Bednarek, J. Adamowski, M.B. Tavernier, E. Anisimovas, and F.M. Peeters, Eur. Phys. J. D 28, 373 (2004).

${ }^{25}$ K. Maki and X. Zotos, Phys. Rev. B 28, 4349 (1983).

${ }^{26}$ R. Price, X. Zhu, S. Das Sarma, and P.M. Platzman, Phys. Rev. B 51, 2017 (1995).
${ }^{27}$ M. Polini, M.K. Moulopolous, B. Davoudi, and M.P. Tosi, Phys. Rev. B 65, 165306 (2002).

${ }^{28}$ J. Kainz, S.A. Mikhailov, A. Wensauer, and U. Rössler, Phys. Rev. B 65, 115305 (2002).

${ }^{29}$ B. Szafran, S. Bednarek, and J. Adamowski, Phys. Rev. B 67, 045311 (2003); J. Phys.: Condens. Matter 15, 4189 (2003).

${ }^{30}$ M.B. Tavernier, E. Anisimovas, F.M. Peeters, B. Szafran, J. Adamowski, and S. Bednarek, Phys. Rev. B 68, 115310 (2003).

${ }^{31}$ K. Jauregui, W. Häusler, and B. Kramer, Europhys. Lett. 24, 581 (1993).

${ }^{32}$ F.M. Peeters, Phys. Rev. B 42, 1486 (1990).

${ }^{33}$ S.R.E. Yang and A.H. MacDonald, Phys. Rev. B 66, 041304 (2002).

${ }^{34}$ This is similar to the equivalent system of vortices in superconducting mesoscopic squares. See, e.g., B.J. Baelus and F.M. Peeters, Phys. Rev. B 65, 104515 (2002).

${ }^{35}$ L.F. Chibotaru, A. Ceulemans, M. Lorenzini, and V.V. Moshchalkov, Europhys. Lett. 63, 159 (2003).

${ }^{36}$ The $\pi / 2$ rotation is not an observable and the average value of its operator is gauge dependent.

${ }^{37}$ Although in the plots in Figs. 25, 28, and 29 Wigner molecules are pinned at a similar orientation small angular differences can be noticed [e.g., the cross formed of the position of electrons in Fig. 29(a) is slightly tilted to left and in Fig. 29(b) to right]. We left these differences uncorrected to show that the positions of the charge maxima do not coincide with the position of centers, fixed at the same angles in all these plots.

${ }^{38}$ B. Reusch and H. Grabert, Phys. Rev. B 68, 045309 (2003).

${ }^{39}$ S. Tarucha, D.G. Austing, T. Honda, R.J. van der Hage, and L.P. Kouwenhoven, Phys. Rev. Lett. 77, 3613 (1996).

${ }^{40}$ B.T. Miller, W. Hansen, S. Manus, R.J. Luyken, A. Lorke, J.P. Kotthaus, S. Huant, G. Medeiros-Ribeiro, and P.M. Petroff, Phys. Rev. B 56, 6764 (1997).

${ }^{41}$ B. Szafran, S. Bednarek, and J. Adamowski, Phys. Rev. B 61, 1971 (2000); 65, 035316 (2002); 67, 115323 (2003). 